\title{
Limits to solar cycle predictability: Cross-equatorial flux plumes
}

\author{
R. H. Cameron ${ }^{1}$, M. Dasi-Espuig ${ }^{1}$, J. Jiang ${ }^{2}$, E. Işsk ${ }^{3}$, D. Schmitt ${ }^{1}$, and M. Schüssler ${ }^{1}$ \\ 1 Max-Planck-Institut für Sonnensystemforschung, 37191 Katlenburg-Lindau, Germany \\ e-mail: cameron@mps.mpg.de \\ 2 Key Laboratory of Solar Activity, National Astronomical Observatories, Chinese Academy of Sciences, Beijing 100012, PR China \\ 3 Department of Physics, Faculty of Science \& Letters, Istanbul Kültür University, Ataköy Campus, Bakırköy 34156, Istanbul, Turkey
}

Received 28 May 2013 / Accepted 8 August 2013

\begin{abstract}
Context. Within the Babcock-Leighton framework for the solar dynamo, the strength of a cycle is expected to depend on the strength of the dipole moment or net hemispheric flux during the preceding minimum, which depends on how much flux was present in each hemisphere at the start of the previous cycle and how much net magnetic flux was transported across the equator during the cycle. Some of this transport is associated with the random walk of magnetic flux tubes subject to granular and supergranular buffeting, some of it is due to the advection caused by systematic cross-equatorial flows such as those associated with the inflows into active regions, and some crosses the equator during the emergence process.

Aims. We aim to determine how much of the cross-equatorial transport is due to small-scale disorganized motions (treated as diffusion) compared with other processes such as emergence flux across the equator.

Methods. We measure the cross-equatorial flux transport using Kitt Peak synoptic magnetograms, estimating both the total and diffusive fluxes.

Results. Occasionally a large sunspot group, with a large tilt angle emerges crossing the equator, with flux from the two polarities in opposite hemispheres. The largest of these events carry a substantial amount of flux across the equator (compared to the magnetic flux near the poles). We call such events cross-equatorial flux plumes. There are very few such large events during a cycle, which introduces an uncertainty into the determination of the amount of magnetic flux transported across the equator in any particular cycle. As the amount of flux which crosses the equator determines the amount of net flux in each hemisphere, it follows that the cross-equatorial plumes introduce an uncertainty in the prediction of the net flux in each hemisphere. This leads to an uncertainty in predictions of the strength of the following cycle.
\end{abstract}

Key words. Sun: surface magnetism

\section{Introduction}

The large-scale field of the Sun, as measured at the surface, reverses roughly every 11 years. A signature of the reversal is the change of sign of the net flux in the southern and northern hemispheres, and the reversal of the polarity orientation of emerging bipoles in each hemisphere according to Hale's law. Application of Stokes' theorem to the induction equation shows that the change in the net magnetic flux through the solar surface of the northern hemisphere is equal to the amount of magnetic flux transported across the equator - being the boundary of the northern hemisphere surface (Durrant et al. 2004).

On the Sun's surface the (molecular) diffusion is small, and the transport is almost entirely due to advection by either largescale systematic flows (such as the Sun's differential rotation and meridional circulation) or small-scale turbulent flows (such as are associated with granulation and supergranulation) - the latter flows shuffle the field in random directions and can be treated as a diffusive process. The transport across the equator (at the surface) occurs either by horizontal transport of radial magnetic field across the equator, or by the radial transport of horizontal field through the solar surface (flux emergence) at the equator.

Cross-equatorial flux transport hence plays a critical role in the evolution of the Sun's large-scale magnetic field (for a discussion of it in the context of solar cycle prediction see Petrovay 2010). In models such as the Surface Flux Transport model (for a recent review, see Mackay \& Yeates 2012), or Flux Transport Dynamo model (Choudhuri et al. 1995), the meridional flows are usually assumed to be anti-symmetric about the equatorial plane, and therefore do not transport flux across the equator. Exceptions in this regard include the work of Jiang et al. (2010) and Cameron \& Schüssler (2012) who considered inflows into the active region belts, which can occasionally extend across the equator. Flux can also emerge across the equator, with different polarities in each hemisphere. The relative importance of these different processes in transporting magnetic flux across the equator can be assessed observationally.

In this paper we use the Kitt Peak synoptic magnetograms to estimate the total net flux transported across the equator. We evaluate this as the time derivative of the net flux in the northern hemisphere. We also estimate the amount which is transported by the small-scale random motions (i.e. the amount of magnetic flux transported across the equator by turbulent diffusion). The difference between the two is the amount of flux transported by processes which can not be described using the diffusive approximation. This nondiffusive component is shown to be associated with discrete events in which highly tilted bipolar groups emerge across the equator, or near the equator and some of the flux is then advected across the equator. In this paper we study examples of each type of event.

\section{Examples of cross-equatorial transport}

Our analysis is based on the National Solar Observatory, Kitt Peak synoptic maps of the radial component of the Sun's 


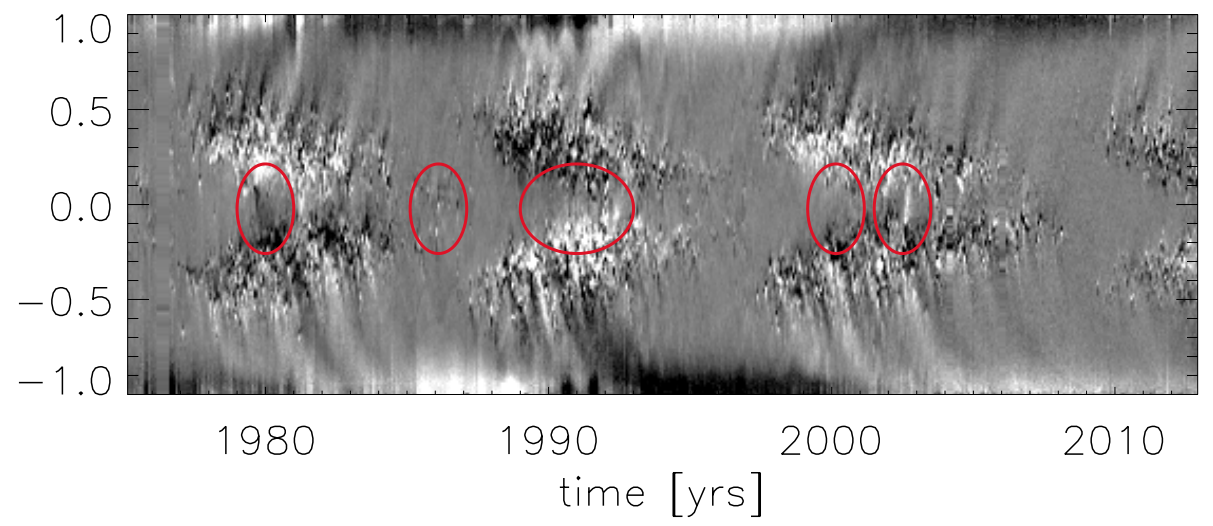

Fig. 1. Longitudinally averaged radial magnetic field as a function of latitude and time from the Kitt Peak Solar Observatory synoptic magnetograms. The gray scale is saturated at $\pm 10 \mathrm{G}$. The red circles illustrate cross-equatorial flux plumes. Black indicates negative magnetic field, white postive. magnetic field, consisting of one map per Carrington rotation (Harvey \& Worden 1998). The maps for Carrington rotations 1625 to 2007 are based on the NSO Vacuum Telescope ${ }^{1}$, thereafter we used the synoptic maps based on the SOLIS telescope ${ }^{2}$.

\subsection{Cross-equatorial flux plumes}

The data gives the radial component of the magnetic field strength as a function latitude, $\lambda$, longitude, $\phi$, and time, $t$. Hereafter we consider the longitudinally averaged field $B_{r}(\lambda, t)$, which is shown in Fig. 1.

Notable features include the wings of the butterfly diagram and the inclined features which extend from the active regions towards the poles. These features were studied by Durrant et al. (2001) and called "flux plumes". Although they are fewer in number, similar inclined features can be seen crossing the equator. The red ellipses in Fig. 1 outline the larger of these events. We call these "cross-equatorial flux plumes", owing to their similarity with the "flux plumes".

\subsection{Emergence across the equator}

Figure 2 shows the evolution of the magnetic field which produces the cross-equatorial flux plume in 1980 circled in Fig. 1. A large bipolar region has emerged with the positive polarity flux in the northern hemisphere and the negative polarity extending to both sides of the equator. The axis of the bipolar pair, the line connecting the centers of the opposite polarities, is inclined at almost 90 degrees to the equator. In this particular case the northsouth orientation of the bipolar pair is opposite to that given by Joy's law for this cycle. A second event that occurred in 1986, in the declining stages of cycle 21, is shown in Fig. 3. Again we have flux emergence across the equator; however in this case the north-south alignment is in accordance with Joy's law. The latitudinal alignment of the bipolar groups is important because it determines whether negative or positive flux is transported into the northern hemisphere. For cycle 21, transporting positive flux into the northern hemisphere acts to weaken the net flux in each hemisphere at the subsequent minimum, whereas transporting negative flux enhances the net flux at minimum. The two events therefore mostly cancel each other for this cycle, as will be discussed in Sect. 4. There are several weak cross-equatorial flux plumes around 1990, before another prominent event occured in 2002.

\footnotetext{
1 The NSO Vacuum Telescope data were obtained from ftp://nsokp.nso.edu/kpvt/synoptic/

2 The SOLIS data was obtained from ftp://solis.nso.edu/ synoptic/level3/vsm/merged/carr-rot/
}

\subsection{Emergence near the equator}

A second type of event is shown in Fig. 4. Here a weaker, but still highly tilted, bipolar region emerges close to the equator. Both polarities emerge in the southern hemisphere, with the negative flux being closer to the equator. Negative flux is transported across the equator, presumably driven by cross-equatorial flows. This leads to a substantial amount of flux crossing the equator.

\section{Measuring the cross-equatorial fluxes}

The calculation of the cross-equatorial flux transport of magnetic flux is discussed by Durrant et al. (2004), who also estimated the diffusive component of the cross-equatorial flux transport during cycle 22 . The net (signed) magnetic flux in the northern hemisphere is given by

$F^{\mathrm{NH}}=\int_{\mathrm{NH}} B_{r} \mathrm{~d} A$,

and in the southern hemisphere by

$F^{\mathrm{SH}}=\int_{\mathrm{SH}} B_{r} \mathrm{~d} A$.

Because $\boldsymbol{\nabla} \cdot \mathbf{B}=0$ these must satisfy $F^{\mathrm{NH}}=-F^{\mathrm{SH}}$. To reduce the noise we define $F=\left(F^{\mathrm{NH}}-F^{\mathrm{SH}}\right) / 2$, i.e. $F$ is an estimate of the flux in the northern hemisphere based on observations over both hemispheres. The net magnetic flux transport across the equator at the solar surface is then determined by $\mathrm{d} F / \mathrm{d} t$.

Because we are numerically evaluating the time derivative, the measured cross-equatorial transport is noisy. One obvious source of noise is the yearly apparent modulation of the polar fields in Fig. 1, which is an artifact introduced by the variation of the solar $B$-angle due to the inclination of the solar rotation axis to the ecliptic. By averaging over 13, 27, or 54 Carrington rotations (approximately 1, 2, and 4 years, respectively) this source of noise is substantially reduced. The black lines in the left-hand panels of Fig. 5 show the time history of $\mathrm{d} F / \mathrm{d} t$.

We also estimate the amount of cross-equatorial flux transport which is due to diffusion of magnetic flux across the equator. We consider the turbulent diffusion describing the random walk of magnetic features associated with supergranulation, averaging the magnetic field over supergranular scales using a box-car filter with a width of $24 \mathrm{Mm}$. We then calculate the latitudinal gradient of the magnetic field at the equator using centered finite differences and estimate the diffusive cross-equatorial flux transport as

$\left(\frac{\mathrm{d} F}{\mathrm{~d} t}\right)_{\text {diffusive }}=-\left.2 \pi \eta_{\text {turb }} \frac{\partial B_{r}}{\partial \lambda}\right|_{\lambda=0}$, 
R. H. Cameron et al.: Limits to solar cycle predictability: Cross-equatorial flux plumes
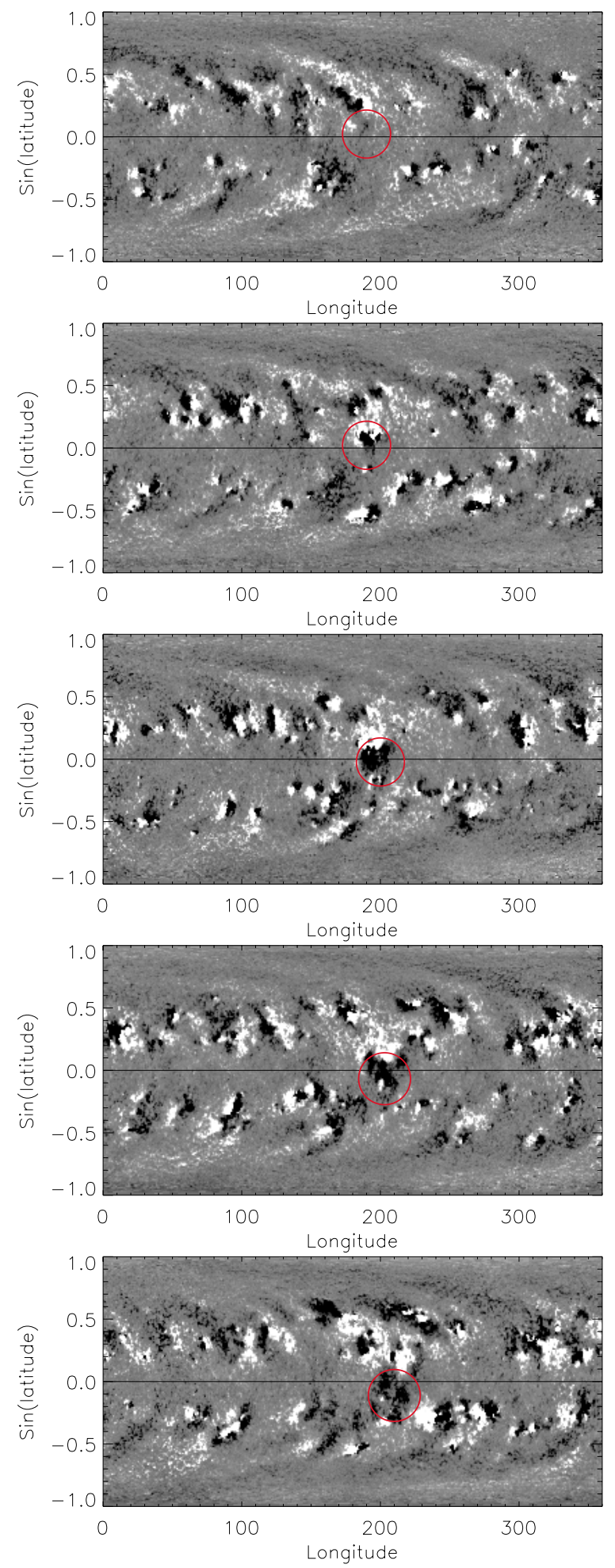

Fig. 2. Kitt Peak synoptic magnetograms for Carrington rotations 16841688 (starting dates: 1979, July 17, August 13, September 9, October 6, November 3) are shown. The red ellipse outlines an example of nondiffusive cross-equatorial transport of magnetic flux. In this case the negative flux of a highly tilted bipolar region emerges across the equator.

where $\lambda$ is latitude and we assume $\eta_{\text {turb }}=250 \mathrm{~km}^{2} \mathrm{~s}^{-1}$ (Cameron et al. 2010). This diffusive component of the cross equatorial flux transport is shown by the red lines in the left-hand panels of Fig. 5. Explicitly, it is the amount of flux carried across the equator by diffusion: how the flux arrives near the equator
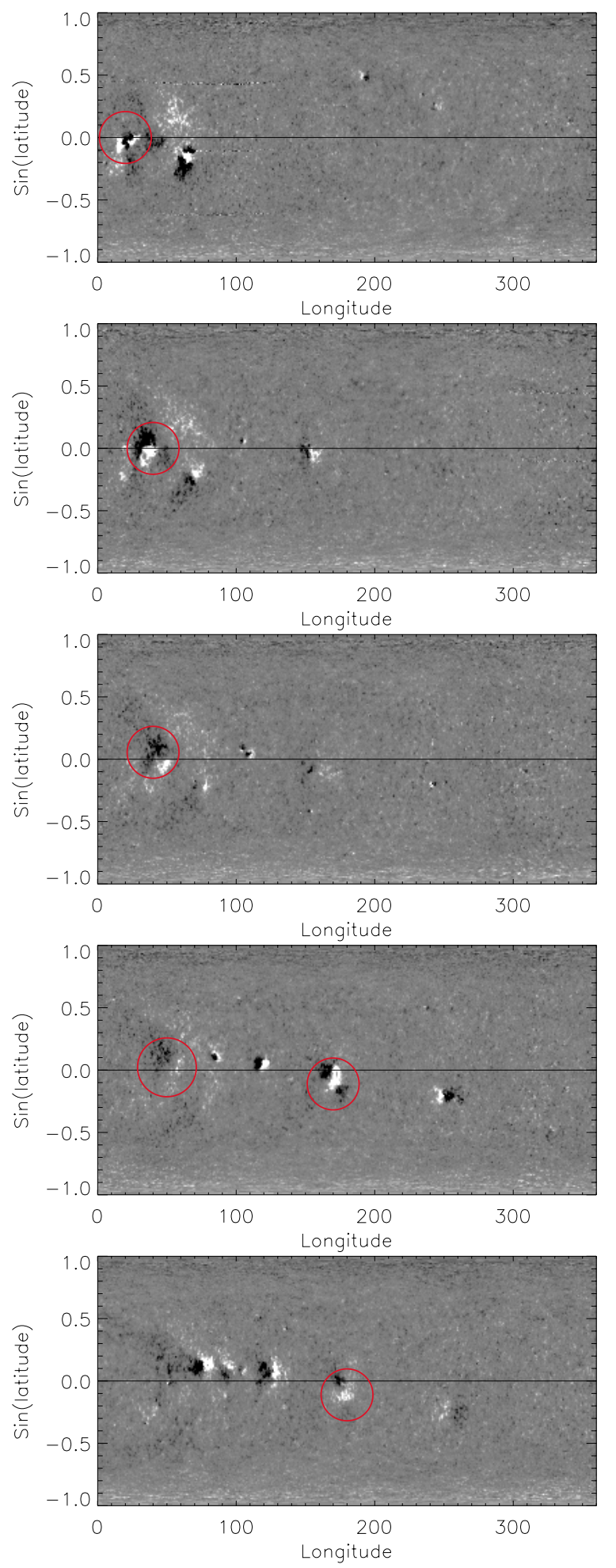

Fig. 3. Kitt Peak synoptic magnetograms for Carrington rotations 17711775 (starting dates: 1986, January 14, February 10, March 9, April 6, May 3). The red circle outlines a magnetic bipole which emerges near the equator. Because it is highly tilted, the positive magnetic flux is almost entirely in the southern hemisphere and the negative flux is in the northern hemisphere. A similar, but weaker, event with a smaller tilt angle occurs towards the end of this event.

(before being transported across the equator by diffusion) will in general involve a mixture of emergence, advection and diffusion. 

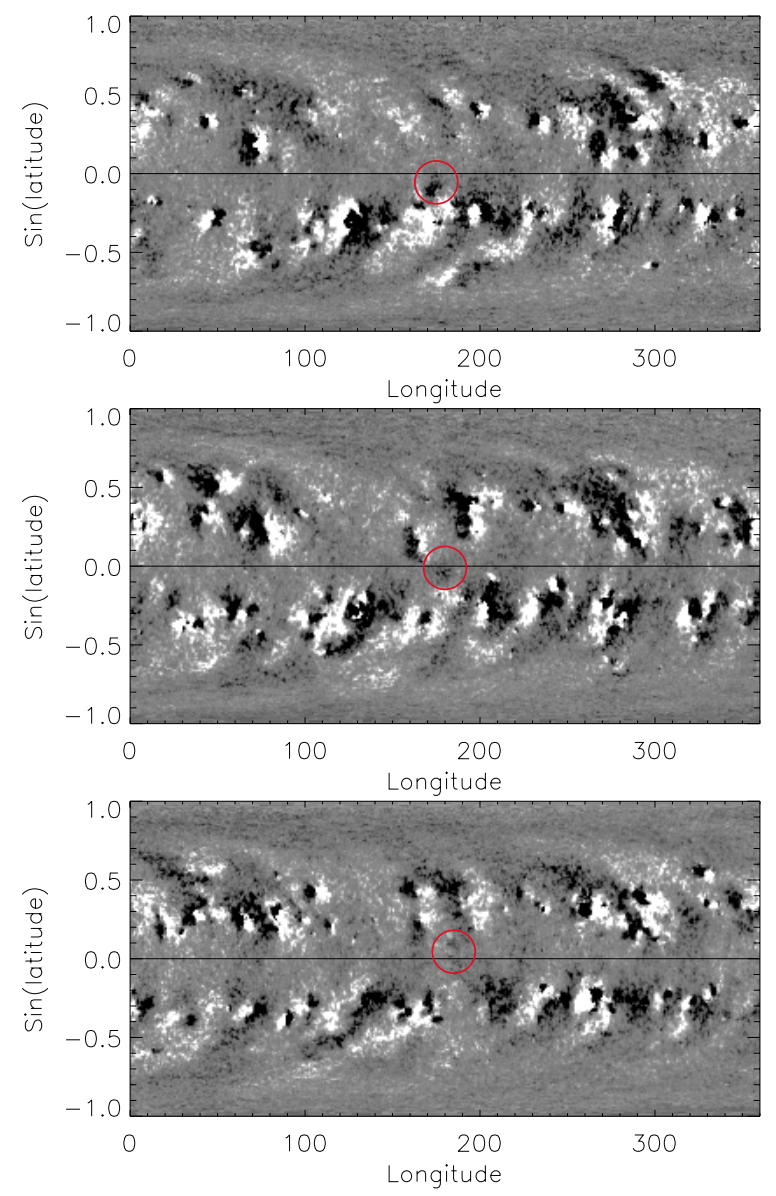

Fig. 4. Kitt Peak synoptic magnetograms for Carrington rotations 19601962 (starting dates: 2000, February 25, March 23, April 19). The red circle outlines negative flux which has emerged near the equator as part of a highly tilted bipolar group. In the course of its evolution, the negative flux is transported across the equator.

\section{Relation of polar flux and net flux in each hemisphere at minima}

So far our discussion has concentrated on the net flux in each hemisphere. The more common precursors used to make predictions of solar activity are the polar fields and open heliospheric flux at solar minimum. Figure 6 shows the contribution of each latitude to the net flux in each hemisphere for the last four minima (those for which observations are available). At solar minimum most of the net flux in each hemisphere is concentrated near the poles: on average over $78 \%$ of the flux in each hemisphere is above $60^{\circ}$ latitude. The variation in the net flux in each hemisphere is thus a major factor in determining the strength of the polar fields at minimum. This is because the by the time that the activity has reached its minimum, the magnetic field distribution has had sufficient time to move toward an equilibrium for which the transport due to advection by the meridional flow towards the poles is balanced by the diffusion of field away from the pole. The relative contributions over the last four cycles do show some variability, but this is substantially less than the variability of the cycle strengths. Hence most of the cycle-to-cycle variability in the polar field strengths is coming from changes in the net flux in each hemispere. We similarly expect the open flux at minimum to be highly correlated with the net flux in each hemisphere.

\section{Discussion}

Comparison of the black and red curves on the left panels of Fig. 5 show that most of the net flux transport across the equator is represented well by diffusion with $\eta_{\text {turb }}=250 \mathrm{~km}^{2} \mathrm{~s}^{-1}$. This value is in accordance with observations (see Table 6.1 Schrijver \& Zwaan 2000), simulation (Cameron et al. 2011), and also with what is required to reproduce the evolution of the Sun's largescale magnetic field using the surface flux transport model with a meridional flow profile consistent with observations (Cameron et al. 2010). While the curves are similar, there are also differences which represent the nondiffusive component of crossequatorial flux transport. The examples of cross-equatorial flux plumes discussed in Sects. 2.2 and 2.3 are clearly associated with such nondiffusive transport. Figure 5 also indicates that there are more cross-equatorial flux plumes than the examples discussed, yet these events are among the most prominent. We note that we chose some of the examples based upon their signature in Fig. 5, although they only have a weak signature in Fig. 1. We also comment that some apparently strong cross-equatorial flux plumes in the magnetic butterfly diagram, such as the event in 2002, show only a weak signature in Fig. 5. In this case, the positive polarity flux plume is preceded by a weaker but longer lived negative polarity flux plume. The time averaging then smoothes these two, erasing the signature of both events.

The impact of the cross-equatorial flux plumes can be seen clearly in the evolution of the net flux in the northern hemisphere, $F$, shown in the right panels of Fig. 5. The amount of flux in the northern hemisphere that, at any given time, which was carried across the equator by diffusion alone can then be calculated as $\int(\mathrm{d} F / \mathrm{d} t)_{\text {diffusive }} \mathrm{d} t+F_{0}$. The value of $F_{0}$ is a timeindependent offset to the net flux in each hemisphere. If the transport were only diffusive, then a single offset (the amount of flux in the northern hemisphere at the start of the time series $F_{0}=2 \times 10^{22} \mathrm{Mx}$ ) would make the curve for the diffusive transport, (shown by the green curve) match the total flux transport (black curve) throughout the entire period. Instead it is only consistent with the total transport until about 1980. At this time there is substantial cross-equatorial transport due to the event shown in Fig. 2. The net flux in the northern hemisphere, which has not yet reversed, is strengthened. This transport is nondiffusive, and so the evolution of the net flux in the northern hemisphere no longer follows the purely diffusive green curve. The evolution after 1980 is again mostly diffusive - the field evolves along the red curve in Fig. 5 which gives the evolution from $F_{0}=4 \times 10^{22} \mathrm{Mx}$ being the sum of the original $F_{0}=2 \times 10^{22} \mathrm{Mx}$ and the $2 \times 10^{22} \mathrm{Mx}$ injected from the nondiffusive transport. This red curve is inconsistent with the evolution before 1980, and has a mean which is higher than the observations. It does however match the observed evolution from 1980 until the event shown in Fig. 3 occurs in 1986 and the net flux in the northern hemisphere drops (nondiffusively) by about $2 \times 10^{22} \mathrm{Mx}$ (back to the green curve). In this particular case, the two events thus cancel each other and the net result is similar to purely diffusive transport. Thereafter, the evolution is again mainly due to diffusion until 1990. There is then a period when a number of weak cross-equatorial positive magnetic plumes can be seen crossing the equator in the magnetic butterfly diagram. Although these plumes individually do not carry much flux, their combined effect is substantial and the evolution switches again to follow the red curve. From 1992 to 2000 the evolution is roughly in accordance with purely diffusive transport. In 2000 another event transports flux across the equator and the evolution switches back to mostly 

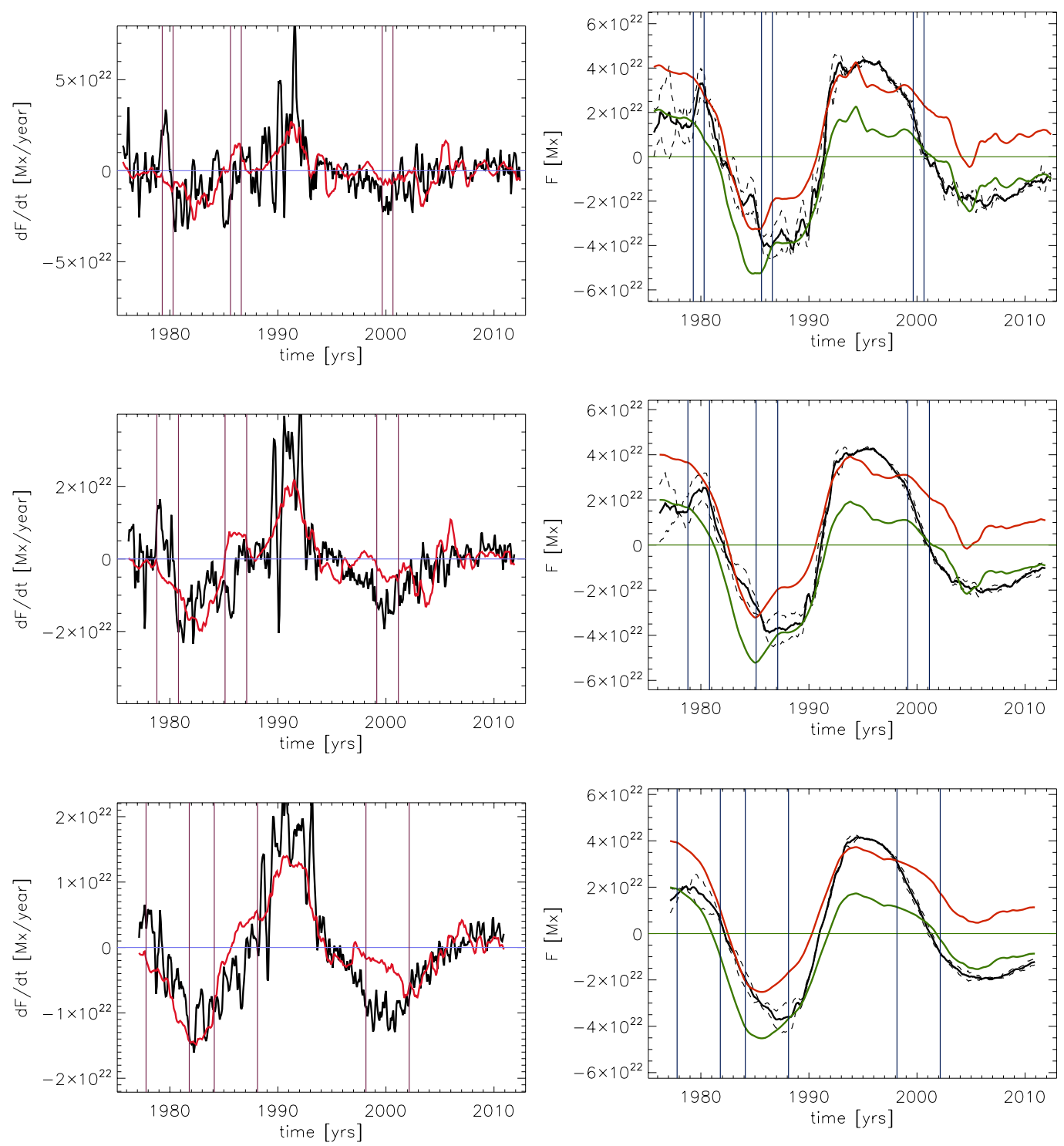

Fig. 5. Net cross equatorial transport of magnetic flux as a function of time (left panels). The black curves show the total net flux transport, $\mathrm{d} F / \mathrm{d} t$ averaged over 1 year (top panel), 2 years (middle panel), and 4 years (bottom panel). The red curves show the diffusive component of the flux transport $\frac{\mathrm{d} F}{\mathrm{~d} t}$ diffusive , see Eq. (3). The right panels show $F^{\mathrm{NH}}$, the magnetic flux in the northern hemisphere, and $-F^{\mathrm{SH}}$, the negative of the magnetic flux in the southern hemisphere (dashed curves), as well as their mean (solid black curve). For comparison the time integral of the diffusive flux is shown in red (assuming a starting value of $4 \times 10^{22} \mathrm{Mx}$ in 1976) and green (assuming a starting value of $2 \times 10^{22} \mathrm{Mx}$ ). The intervals outlined by the vertical lines indicate the periods affected by the three events shown in Figs. 2-4, respectively.

diffusive transport at about the level of the green curve with $F_{0}=2 \times 10^{22} \mathrm{Mx}$.

Each of the cross-equatorial transport events studied here transports about $2 \times 10^{22} \mathrm{Mx}$ of flux across the equator (presumably due to chance and because we focussed on the largest events). Consistent with the synoptic magnetograms, this is a significant fraction of the total unsigned flux associated with a large active region (Schrijver \& Zwaan 2000, Table 5.1, gives $1 \times 10^{22}$ to $6 \times 10^{22} \mathrm{Mx}$ as the range for the unsigned flux of a large active region). The diffusive flux integrated over a cycle can be estimated from Fig. 5 to be about $6 \times 10^{22} \mathrm{Mx}$, which is only about 3 times larger than the flux carried by the largest of the observed cross-equatorial flux plumes. It follows that a single cross-equatorial flux plume introduces a change in the total flux transported across the equator during a cycle of approximately $\pm 30 \%$. The effect on the polar fields is about twice this, that is a change of $\pm 60 \%$. This is because the flux which crosses the equator first cancels the existing polar fields, and then reverses them. The amount of flux required to cancel the existing flux is independent of the total amount of flux which is transported across the equator. The flux which has been transported across the equator only contributes to the reversed net hemispheric flux after the (fixed) amount of flux which is required to cancel the old flux is surpassed.

In the cases studied here, we have events which weaken the net hemispheric flux at the subsequent minimum (the crossequatorial flux plume in 1980) as well as those which strengthen it (the events in 1986 and 2000). We also have an example where the event occurred before maximum, as well as one which occurred near (but before) the minimum. Because the events cancel in some cycles, the net flux carried by the plumes in the cycles studied here is about $\pm 10^{22} \mathrm{Mx} /$ cycle. A failure to account for 


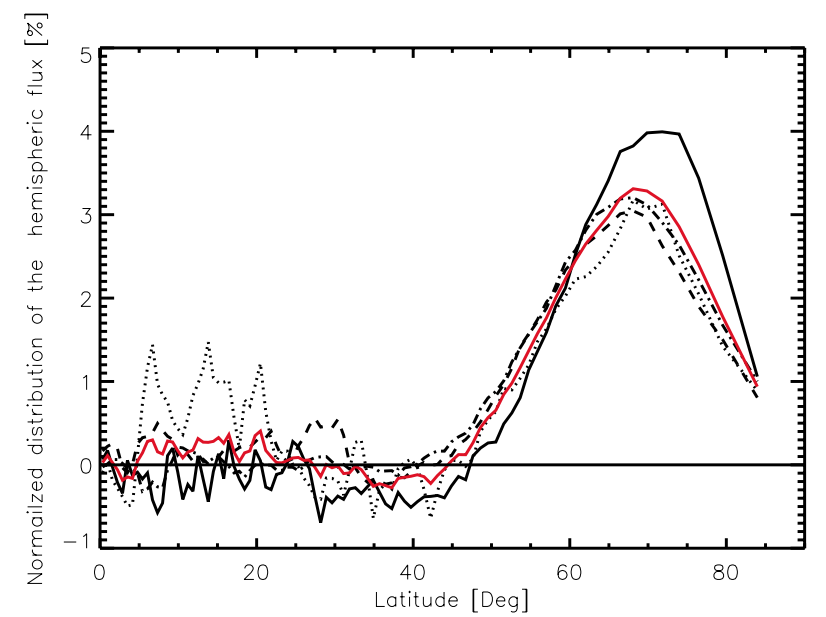

Fig. 6. Normalized distribution of the net flux in each hemisphere per degree as a function of latitude for the minimum between cycles 23 to 24 (solid black), cycle 22 to 23 (dash-dot), 21 to 22 (dashed) and 20 to 21 (dotted). In each case the fluxes have been averaged over 2 years centered on the minimum. The red curve shows the average over all the cycles.

this flux in, for example, surface flux transport models will cause the model to undergo a random walk away from the observed flux. For modeling cycles 15 to 21, as for example in Cameron et al. (2010), where the tilt angles of individual bipolar groups is not available and a (cycle-dependent) Joy's law is assumed, the RMS error introduced by not modeling the types of events is $9.8 \times 10^{21} \mathrm{Mx}$. This was calculated assuming a random walk of $\pm 10^{22} \mathrm{Mx} /$ cycle and noting that the initial field strength of the model was a free parameter. This is to be compared with the range of fluxes, from $2.5 \times 10^{22} \mathrm{Mx}$ to $6 \times 10^{22} \mathrm{Mx}$, for the open flux at minima reported by Lockwood (2003). Thus SFT simulations for a few solar cycles are justified, even if they neglect the cross-equatorial plumes studied here. However the fact that the error is associated with a random walk means that the RMS error increases with the number of cycles simulated, and occassionally the errors introduced by a single cycle will be important. Because the number of large events is small, we are unable to comment further on their statistics.

\section{Conclusion}

The main result of this work is that a single cross-equatorial flux plumes can affect the net hemispheric flux of the following minima by up to $60 \%$. Furthermore, whether the effect is to enhance or weaken the net flux depends on whether the tilt is positive or negative. The number of cross-equatorial flux plumes studied here is too small to evaluate whether there is a preference for constructive or destructive events. Therefore, any prediction of the net flux in each hemisphere is subject to a large uncertainty. Moreover, the strong correlation between the minima of the geomagnetic $a a$ indices (a proxy for the Sun's axial dipole moment) and the amplitude of the next cycle (Wang \& Sheeley 2009), indicates that this uncertainty should carry over directly into an uncertainty in predictions of the level of the Sun's activity (e.g. Schatten et al. 1978; Jiang et al. 2007; Yeates 2013).

Not every cycle may have such a large cross-equatorial flux plume, and some cycles might have several smaller flux plumes which partially cancel each other. Nonetheless these events probably will have a substantial random impact on the strength of the net hemispheric flux. In rare instances it is possible that the flux transport across the equator could be effectively reduced by $50 \%$ and lead to no net flux during the subsequent minimum. This might be a scenario for the origin of grand minima in the Babcock-Leighton model of the solar dynamo. In any case even single flux plumes, such as occurred in cycles 21,22 and 23 , can have a large impact on the evolution of the polar fields.

Acknowledgements. NSO/Kitt Peak data used here are produced cooperatively by NSF/NOAO, NASA/GSFC, and NOAA/SEL. This work utilizes SOLIS data obtained by the NSO Integrated Synoptic Program (NISP), managed by the National Solar Observatory, which is operated by the Association of Universities for Research in Astronomy (AURA), Inc. under a cooperative agreement with the National Science Foundation. J.J. acknowledges financial support from the National Natural Science Foundations of China through grant 11173033.

\section{References}

Cameron, R., Vögler, A., \& Schüssler, M. 2011, A\&A, 533, A86 Cameron, R. H., \& Schüssler, M. 2012, A\&A, 548, A57

Cameron, R. H., Jiang, J., Schmitt, D., \& Schüssler, M. 2010, ApJ, 719, 264

Choudhuri, A. R., Schussler, M., \& Dikpati, M. 1995, A\&A, 303, L29

Durrant, C. J., Kress, J. M., \& Wilson, P. R. 2001, Sol. Phys., 201, 57

Durrant, C. J., Turner, J. P. R., \& Wilson, P. R. 2004, Sol. Phys., 222, 345

Harvey, J., \& Worden, J. 1998, in Synoptic Solar Physics, eds. K. S. Balasubramaniam, J. Harvey, \& D. Rabin, ASP Conf. Ser., 140, 155

Jiang, J., Chatterjee, P., \& Choudhuri, A. R. 2007, MNRAS, 381, 1527

Jiang, J., Işik, E., Cameron, R. H., Schmitt, D., \& Schüssler, M. 2010, ApJ, 717, 597

Lockwood, M. 2003, J. Geophys. Res., 108, 1128

Mackay, D., \& Yeates, A. 2012, Living Rev. Sol. Phys., 9, 6

Petrovay, K. 2010, Living Rev. Sol. Phys., 7, 6

Schatten, K. H., Scherrer, P. H., Svalgaard, L., \& Wilcox, J. M. 1978, Geophys. Res. Lett., 5, 411

Schrijver, C. J., \& Zwaan, C. 2000, Solar and Stellar Magnetic Activity, Camb. Astrophys. Ser., 34

Wang, Y.-M., \& Sheeley, N. R. 2009, ApJ, 694, L11

Yeates, A. R. 2013, Sol. Phys., in press 